\title{
1 Host sexual dimorphism affects the outcome of within-host pathogen competition
}

3 Stephen A.Y. Gipson ${ }^{1,2}$; Luis Jimenez ${ }^{1}$; and Matthew D. Hall ${ }^{1}$

4 1. School of Biological Sciences and Centre for Geometric Biology, Monash University, Melbourne,

5 Victoria 3800, Australia

6 2. stephen.gipson@monash.edu

8 Keywords: sexual dimorphism; co-infection; pathogen genetic variation; virulence evolution;

9 Daphnia magna; Pasteuria ramosa

\section{Abstract}

Natural infections often consist multiple pathogens of the same or different species. In multiple infections, pathogens compete for access to host resources and fitness is determined by how well a pathogen can reproduce compared to its competitors. Given the propensity for males and females to exhibit variation in pathogen-induced reduction in lifespan or fecundity, we explore how host sex may modulate the competitive ability of pathogens, potentially favouring the transmission of different pathogen genotypes. Using the Daphnia magna - Pasteuria ramosa model system, we exposed male and female hosts to either a single genotype infection or coinfections consisting of two pathogen genotypes of varying levels of virulence, measured as pathogen-induced reduction in host lifespan. We found that co-infections within females generally favoured the transmission of the more virulent pathogen genotype. Conversely, co-infections within male hosts resulted in equal transmission of competing genotypes, or favoured the transmission of the less virulent pathogen genotype in treatments where it established prior to the more virulent competitor. These results suggest that sex is a form of host heterogeneity which may influence the evolution of virulence within co-infection contexts and that one sex may be a reservoir for pathogen genetic diversity in nature. 


\section{Introduction}

29

30

Due to the ubiquity of pathogens in natural populations, individuals are usually infected with more than one type of pathogen or multiple strains of a single pathogen (Read and Taylor 2001; Rigaud et al. 2010; Balmer and Tanner 2011). In these multiple infection contexts, pathogens compete for access to host resources and the most fit competitor is often the one which induces the greatest impacts on host lifespan or fecundity (reviewed in Alizon et al. 2013). It is in this relationship between host exploitation and pathogen fitness that the predictions for multiple infections vary from those of single infections. While prudent pathogen exploitation leads to the highest pathogen fitness in single infection contexts, represented by a trade-off between pathogen reproduction and host harm (Bull 1994; Frank 1996; Alizon et al. 2009), a pathogen's ability to acquire more resources than competing strains largely dictates relative pathogen fitness in multiple infections. Yet, host populations are rarely homogeneous and pathogens will encounter hosts that vary in characteristics such as age at exposure, nutritional background, or genotype (Wolinska and King 2009). Consequentially, the relative fitness (Hodgson et al. 2004; Izhar et al. 2015; Louhi et al. 2015) as well as the competitive exclusion or coexistence of co-infecting pathogens (de Roode et al. 2004) will depend not only on the virulence of the pathogens involved but also on the characteristics of their hosts.

A near ubiquitous source of host heterogeneity with the potential to modify the outcome of within-host pathogen competition are the differences between male and female hosts. Exhibiting differences in life-history strategies, physiology, or behaviour (Parker 2006; Schärer et al. 2012), the sexes vary in many characteristics that can influence pathogen exploitation (Duneau et al. 2012; Gipson and Hall 2016). For example, the sexes often vary in their relative immune investment (Rolff 2002; Zuk 2009) with one sex experiencing increased prevalence or severity of infection across many species (Poulin 1996; Schalk and Forbes 1997; McCurdy et al. 1998; Sheridan et al. 2000; Zuk 2009; Cousineau and Alizon 2014). The sexes may also represent hosts of varying exploitative potential due to differences in lifespan or physiological traits such as body size (Christe et al. 2007; Duneau and Ebert 2012; Thompson et al. 2017; Gipson and Hall 2018). Ultimately, these differences can result in infection having sex-specific effects on host fecundity or lifespan (see Table 1, Cousineau and Alizon 2014; Giefing-Kröll et al. 2015; Klein and Flanagan 2016), characteristics which drive within-host competition between pathogens (Alizon et al. 2013; Bashey 2015). Yet, it remains largely unexplored how differences between male and female hosts 
may influence the nature of within-host pathogen competition (but see Thompson et al. 2017) and potentially favour the transmission of different pathogen genotypes.

Within-host competition between pathogens can take a number of forms including: 1)

Exploitation, where pathogens compete over a limited host resource pool; 2) Apparent, where the immune response stimulated by one pathogen indirectly inhibits competitors; or 3) Interference, where pathogen reproduction directly inhibits competitors either through prior establishment or competition over physical space (Mideo 2009; Balmer and Tanner 2011; Alizon et al. 2013; Bashey 2015; Cressler et al. 2016) - each form having different predicted outcomes for virulence evolution. Resource competition, for example, may cause pathogens to overexploit their host, leading to increased virulence (Chao et al. 2000; de Roode et al. 2005a); yet competing pathogens may interfere with one another or plastically adjust their rate of exploitation, leading to decreased virulence in these competition contexts (Massey et al. 2004; Choisy and de Roode 2010;

Eswarappa et al. 2012). Inhibition may also extend to infections where one genotype establishes prior to the other; often favouring the earlier establishing pathogen regardless of their virulence characteristics (de Roode et al. 2005a; Eswarappa et al. 2012). Yet, as the most fit competitor will depend on the specific characteristics of its host (de Roode et al. 2004; Hodgson et al. 2004; Råberg et al. 2006; Ben-Ami et al. 2008; Ben-Ami and Routtu 2013; Izhar et al. 2015; Louhi et al. 2015), heterogeneity may be an important factor in determining the virulence of co-infections.

Within each form of pathogen competition exist unexplored opportunities for heterogeneity due to host sex to modulate the way pathogens exploit their host and influence the outcome of coinfection. The previously discussed effects of host sex on fecundity and lifespan, for example, may affect the relative competitive ability of pathogens by impacting on patterns of pathogen virulence. Additionally, physiological differences between the sexes may cause one sex to have less resources or physical space for a pathogen to exploit (Duneau and Ebert 2012; Thompson et al. 2017; Gipson and Hall 2018), potentially intensifying the competition between pathogens over host resources, or limiting the potential for pathogens to establish in previously infected hosts. The sexes may also vary in their immune response upon infection which may affect subsequent pathogen establishment. Given the propensity for males and females to vary in ways relevant to pathogen competition, sex may represent a further source of host heterogeneity impacting on pathogen transmission within co-infection contexts. 
In this study, we use the Daphnia magna - Pasteuria ramosa model system to assess the impact that host sex has on the outcome of multiple infection. In this system, male Daphnia are smaller, exhibit shorter lifespans, are more resistant to infection, experience lower rates of infectioninduced mortality, and allow for less production of pathogen spores; collectively suggesting that they are a more difficult resource to exploit than females (Duneau et al. 2012; Thompson et al. 2017; Gipson and Hall 2018). In nature, Daphnia may be infected with as many as eight genotypes of $P$. ramosa (Mouton et al. 2007) and have consequentially been used to study the evolution of virulence and competition between pathogens within co-infection contexts (Ben-Ami et al. 2008; Andras and Ebert 2013; Ben-Ami and Routtu 2013; Izhar et al. 2015). Co-infections in this system often resemble the virulence of the most virulent competitor in isolation and favour the transmission of more virulent genotypes except for situations in which the less virulent genotype establishes first (Ben-Ami et al. 2008; Ben-Ami and Routtu 2013). Yet co-infections play out differently between males and females, where females exhibit pathogen virulence and reproduction intermediate to that of the competing pathogens in isolation, whereas the outcome within males is more variable (Thompson et al. 2017). Remaining to be explored though is how these sex-specific patterns of co-infection may impact on the relative fitness among pathogen genotypes and the implications this may have for the maintenance of genetic variation and virulence evolution.

Using pathogen genotypes of known virulence characteristics (Clerc et al. 2015), we exposed genetically identical male and female Daphnia to either a single pathogen genotype or to a coinfection consisting of two pathogen genotypes of varying levels of virulence. We then varied the schedule of these exposures, either exposing hosts a single time, or allowing infection to establish prior to a subsequent exposure. We measured pathogen-induced reduction in lifespan (virulence), overall pathogen spore production (transmission), and the relative spore production of competing pathogen genotypes using microsatellite analysis. Due to the relationship between virulence and pathogen competitive ability, as well as the often observed sex-specific patterns of disease outcome, we make the following predictions: 1 ) the less exploitable sex will suppress variation in pathogen virulence, resulting in similar transmission of competing genotypes; 2 ) the more exploitable sex will allow for pathogens to exhibit variation in virulence, favouring the transmission of the less virulent genotype; and consequentially 3 ) the patterns of sequential coinfections in the more exploitable sex will resemble those of theory and empirical studies (de Roode et al. 2005a; Ben-Ami et al. 2008; Eswarappa et al. 2012), whereas the less exploitable sex 
121 will exhibit similar transmission of each genotype regardless of the relative virulence of the

122 previously established genotype. We discuss the implications of these patterns for the

123 maintenance of pathogen genetic variation and the evolution of virulence.

124 Methods

125 Daphnia magna Straus is a globally distributed freshwater crustacean which produces genetically 126 identical male and female offspring via cyclic parthenogenesis (Ebert 2005). During filter feeding,

127 Daphnia encounter the bacterial pathogen Pasteuria ramosa which reduces the lifespan and 128 fecundity of its host (reviewed in Ebert et al. 2016). P. ramosa is an obligate killing pathogen, 129 transmitting exclusively horizontally after inducing host death. This experiment utilized host 130 genotype HU-HO-2 and novel P. ramosa genotypes C19, C24, and C1. Prior to the experiment, we 131 established a parental generation by isolating juvenile female Daphnia from pre-existing stock 132 cultures and maintaining them in standardized conditions for three generations to minimize 133 maternal effects. Juvenile female Daphnia were raised individually in $60-\mathrm{mL}$ vials filled with $50 \mathrm{~mL}$ 134 of artificial Daphnia medium (ADaM, Klüttgen et al. 1994; modified as per Ebert et al. 1998) and 135 were transferred into fresh ADaM twice weekly. These females were maintained at $20^{\circ} \mathrm{C}$, exposed 136 to a 16-hour light to 8-hour dark cycle, and fed up to 5 million cells of Scenedesmus sp. green algae 137 daily.

\section{Production of experimental animals}

139 Once the third-generation standardized females released their first clutch, they were exposed to a 140 short pulse of the hormone methyl farnesoate $(300 \mu \mathrm{g} / \mathrm{L}$, Product ID: S-0153, Echelon Biosciences, 141 Salt Lake City, Utah) to stimulate the production of genetically identical male and female offspring. 142 Following previously established methods (Thompson et al. 2017), the standardized females were 143 transferred into $60-\mathrm{mL}$ vials filled with $20 \mathrm{~mL}$ of hormone treated $A D a M$ and were transferred into 144 fresh hormone treated ADaM three times weekly. Male and female offspring were collected from 145 the second and third clutches post hormone exposure. This treatment has previously been shown 146 to have no detectible impact on host lifespan and fecundity, nor pathogen transmission and 147 virulence (see Table 3, Thompson et al. 2017). 
149 To measure the effect of host sex on the outcome of within-host pathogen competition, as well as 150 how this competition proceeds when one genotype has already established, we randomly exposed 151 males and females to either single infections or co-infections. These exposures were carried out in 152 one of two exposure "schedules": 1) simultaneous exposure occurred once when the individual 153 was five days old or 2) sequential exposure occurred at 5 and 12 days old (see Table 1 for infection 154 design details). All exposures consisted of a 40,000 pathogen spores. In sequential co-infections, 155 the host was exposed to 20,000 spores of one pathogen genotype a week prior to 20,000 spores 156 of the second to allow for prior establishment of infection. In sequential single infections, the host 157 was simply exposed to 20,000 spores at each of the exposure periods by the same genotype. We 158 herein refer to the multiplicity of genotypes and schedule of exposures collectively as "co159 infection treatment."

Three $P$. ramosa genotypes with previously studied disease characteristics were used in this study.

161 When singly infecting female Daphnia, genotype C19 exhibits high virulence and low transmission 162 (average infection duration: 45.29 days; average spore load: 8.56 million spores) whereas genotypes C24 and C1 cause similar infection outcomes, exhibiting lower average virulence and 164 higher average transmission as compared to C19 (Clerc et al. 2015). Co-infections consisted of genotype $\mathrm{C} 19$ and either $\mathrm{C} 24$ or $\mathrm{C} 1$ as these genotype pairings represent similar virulence combinations and were thus predicted to exhibit similar patterns of competitive outcome.

167 Additionally, genotypes C24 and C1 cannot be distinguished using our genetic analyses whereas 168 C19 can be distinguished from C24 or C1, allowing us to determine the relative contribution of each pathogen genotype to the total spore production of co-infected Daphnia (see Genetic 170 analysis section).

17133 individuals of each sex were allocated to each co-infection or uninfected control treatment. In 172 total this experiment consisted of 26 treatments (33 replicates * 2 sex * [3 simultaneous single 173 infections + 3 sequential single infections +2 simultaneous co-infections +2 sequential co174 infections treated with C19 first + 2 sequential co-infections treated with C19 second + 1 175 uninfected control treatment] $=858$ individuals $)$.

\section{Measures of disease characteristics}

177 Survival was checked daily to assess lifespan of control and infected individuals. We calculated 178 virulence by subtracting individual infected male or female lifespans from the average male or 
179 female control lifespan respectively. Upon host death, Daphnia were individually frozen in $500 \mu \mathrm{L}$ 180 of purified water for later determination of infection status and pathogen fitness as measured by 181 overall production of transmission spores. Infection status was assessed by thawing a Daphnia 182 sample, crushing it with a pestle, and noting the presence or absence of mature transmission 183 spores using phase-contrast microscopy. Individuals identified as infected were immediately assessed for overall spore production using an Accuri C6 flow cytometer (BD Biosciences, San Jose, California). The spore load of each infected Daphnia was measured by diluting $10 \mu \mathrm{L}$ of Daphnia sample into $190 \mu \mathrm{L}$ of $5 \mathrm{mM}$ EDTA and loading this dilution into one well of a round-bottomed PPE 96-well plate. Custom gates based on fluorescence (FL3) were used to omit algae cells from the final count and custom gates based on side scatter (SSA) were used to identify only mature spores based on their distinct size and morphology compared to immature spores and animal debris (Ebert et al. 2016). Overall spore load was measured twice per individual and averaged.

\section{Genetic analysis and measures of within-host pathogen competition}

To assess the fitness of co-infecting pathogen genotypes, we performed DNA extractions on coinfected Daphnia and determined the relative contribution of each pathogen genotype using variable number tandem repeats (Mouton et al. 2007). Pathogen genotypes were distinguished using primer sequences $\operatorname{Pr} 1, \operatorname{Pr} 2$, and $\operatorname{Pr} 3$ (Table 2, Mouton et al. 2007) which have been previously used to distinguish P. ramosa isolate P1 from isolates P4 and P5 (Ben-Ami and Routtu 2013) from which P. ramosa clones C19, or C24 and C1 are respectively derived (Luijckx 2012). As clones from isolates P4 and P5 cannot be distinguished using this method, co-infections always consisted of pathogen genotype C19 and C24 or C1.

DNA extractions were performed using the EZNA Tissue DNA kit (Omega Bio-tek, Norcross, Georgia) with a modified protocol based on similar studies assessing the genetic composition of $P$. ramosa infections (Ben-Ami et al. 2008; Andras and Ebert 2013; Ben-Ami and Routtu 2013; Izhar et al. 2015). Immediately after spore counting, the crushed Daphnia samples were pelleted via centrifugation for 3 minutes at 12,205 RCF, supernatant removed, and washed with $1 \mathrm{~mL}$ of double-distilled water. The samples were again centrifuged using the same settings, supernatant removed, and suspended in $200 \mu \mathrm{L}$ lysis buffer and $25 \mu \mathrm{L}$ OB protease. The samples were then homogenized via bead beating with approximately $0.25 \mathrm{~g}$ of $0.1 \mathrm{~mm}$ zirconia beads for 2 minutes ( $1 \times 10$ s, $1 \times 20$ s, and $3 \times 30$ s). Subsequently, samples were incubated in a heat block at $55^{\circ} \mathrm{C}$ for 1 hour before centrifugation at $10^{\circ} \mathrm{C}$ for 15 minutes at 5005 RCF. After collecting the supernatant, 
210 the DNA extraction proceeded as directed by the manufacturer protocol. An optional step of

211 incubating samples for 2 minutes at $70{ }^{\circ} \mathrm{C}$ prior to elution greatly increased DNA yields. Final

212 elution volume was $100 \mu \mathrm{L}$.

213 DNA was amplified via PCR with temperature cycling methods identical to Andras and Ebert

214 (2013). Fragment analysis and genotyping was performed on these PCR products by AGRF

215 (Melbourne, Australia) to determine the size of microsatellite alleles and the strength of their

216 fluorescence (represented by peak height). The peak height ratio of the microsatellite markers was

217 interpreted as the relative proportion of spores produced by each pathogen genotype as

218 described by Ben-Ami et al. (2008); an approach that has also been used to quantify mixed sperm

219 stores (Bussière et al. 2010). This proportion was multiplied by the absolute number of spores

220 produced within the infected host to determine the relative transmission of competing genotypes.

\section{Statistical analyses}

Only 823 of the 858 individuals initially set up for this experiment were used in final analyses as some individuals either died before infection status can be adequately determined (14 days post exposure, Clerc et al. 2015) or were removed due to experimental error. These 823 individuals consisted of 65 uninfected control individuals, 129 exposed but uninfected individuals, 319 individuals infected from single infection treatments, and 310 individuals infected from coinfection treatments of which P. ramosa DNA was extracted from 290 individuals. All statistical analyses were performed in R (version 3.4.1; R Development Team, available at www.rproject.org). Studies of multiple infection commonly explore how the most fit pathogen genotype is related to reduction in host fecundity or lifespan upon infection (reviewed in Alizon et al. 2013; Bashey 2015). Here, we focused on pathogen induced reduction in lifespan as a comparable measure of pathogen virulence between males and females. We then related patterns of virulence to overall pathogen transmission as well as the transmission of individual co-infecting pathogen genotypes within a mixed infection.

We first explored how the relationship between pathogen virulence and overall pathogen fitness changes due to host sex, pathogen co-infection treatment and their interaction using a multivariate analysis of variance (MANOVA Type III, car package, Fox and Weisberg 2011). Then we analysed the effects of sex, pathogen co-infection treatment, and their interaction on pathogen spore production and virulence using full-factorial analyses of variance (white corrected 
ANOVA Type III, car package). Using the eemeans package (https://github.com/rvlenth/emmeans) to perform post-hoc comparisons of the multivariate means for equivalence, we then explored how the relationship between virulence and overall spore production varied due to host sex or either of the two pathogen genotype combinations (C19 and C24 or C19 and C1). Finally, we tested whether the fitness of individual co-infecting pathogen genotypes changed due to host sex, co-infection treatment, or their interaction. To do this we fit a linear mixed model including each co-infection treatment (Ime4 package, Bates et al. 2015) with an individual's unique ID fit as a random effect, an interaction between sex and pathogen genotype as fixed effects, and relative production of spores as the response. We also fit separate mixed models for each co-infection treatment in order to describe which co-infection treatment was driving patterns of significance in the full model.

\section{Results}

The effects of host sex on pathogen virulence, transmission, and their interaction

Our results indicate interactive effects of host sex and patterns of co-infection on the combined influence of pathogen fitness (the total spore production of co-infections or single genotype infections) and pathogen induced reduction in host lifespan (virulence). Across all single and coinfection treatments, the multivariate ANOVA reveals how infections in females lead to higher spore loads and a greater reduction in lifespan than in males (Fig. 1); but that the magnitude of any response to infection is not shared equally between the sexes due to the presence of the interaction term (Table 2). Figure 1 and Table 2 (univariate ANOVA) suggest that this interaction is largely driven by greater variation in spore loads between co-infection treatments occurring in females (Fig. 1a), with pathogens producing between 9.6 million and 5.9 million average spores within females and between 1.9 million and 1.1 million average spores within males. In contrast, females experienced higher levels of virulence than males, but the relative differences in virulence among co-infection treatments was similar between the sexes (Fig. 1b). Collectively this suggests that while pathogens which delay host death produce the greatest number of spores in females, this trade-off is more dampened in males where variation in virulence relates to a much smaller range in pathogen reproduction (Fig. 1).

Despite the difference in the scale of the trade-off between males and females, within each sex the relationship between pathogen transmission and virulence across co-infection treatments 
270 remained qualitatively similar. As expected, in females we found pathogen genotype C19 was 271 more virulent and produced less transmission spores than C24 (Fig. 2a) or C1 (Fig. 2b) in single 272 infection contexts. Similarly, sequential single genotype infections were indistinguishable from 273 simultaneous single infections. Simultaneous co-infections always behaved like the more virulent 274 pathogen in single infection contexts (C19), yet the order of sequential co-infections influenced 275 the relationship between virulence and transmission differently based on the genotype of the 276 competitor. In competitions with between C19 and C1, sequential co-infections always behaved 277 like the genotype that established first (i.e. sequential C19, C1 behaved like simultaneous C19, 278 C19; Fig. 2b). In contrast, the relationship between virulence and transmission of sequential co279 infections were indistinguishable in competitions between C19 and C24 (Fig. 2a). Males exhibited 280 comparatively similar patterns to females, with C19 more virulent and producing less spores than 281 C24 (Fig. 3a) or C1 (Fig. 3b) in single infections; sequential single infections exhibiting similar 282 patterns to simultaneous single infections, sequential co-infections with C24 exhibiting similar 283 patterns regardless of infection order, and sequential co-infections with C1 behaving like the 284 genotype which established first.

The effects of host sex on the relative fitness among co-infecting pathogen genotypes

Finally, we explored how host sex and pathogen genotype collectively influence the relative pathogen fitness within co-infection contexts. We found that an interaction between host sex and pathogen genotype determined relative pathogen fitness (Table 3). Upon examining each pathogen co-infection treatment individually, an interaction between sex and pathogen genotype influencing relative pathogen fitness was detected for every co-infection treatment except for sequential C24, C19 (Table 3). In females, the more virulent genotype (C19) produced the majority of transmission spores in each co-infection context except for when pathogen genotype C24 established first (Fig. 4). Conversely, co-infection in males resulted in equal spore production of the competing genotypes except for when the less virulent genotypes (C24 or C1) established before C19. In both of these cases, prior establishment of the less virulent genotype resulted in C19 being competitively inferior. In general, coinfections within females were represented by the disproportionate transmission of the more virulent pathogen, but this was not observed in males. For example, in sequential infections with C24, pathogen C19 produced $90.4 \%$ of the total spores within female hosts, but only produced $49.8 \%$ of spores within males. 


\section{Discussion}

301 Much of our understanding on how pathogens should exploit hosts and how exploitation 302 strategies should evolve are influenced by studies focusing on single infections. Yet in nature, 303 infection is more likely to co-occur between multiple pathogen genotypes of the same or different 304 species (Read and Taylor 2001; Rigaud et al. 2010; Balmer and Tanner 2011). When multiple 305 pathogens establish within a host, theory predicts that co-infection commonly favours more virulent pathogens through increased competition for host resources (Alizon et al. 2013). However, host heterogeneity may affect the relative fitness among competing pathogens (de Roode et al. 2004; Hodgson et al. 2004; Råberg et al. 2006; Ben-Ami et al. 2008; Ben-Ami and Routtu 2013; Izhar et al. 2015; Louhi et al. 2015) thus the evolution of more virulent pathogens is not necessarily a universal outcome of co-infection (Alizon et al. 2013; Cressler et al. 2016). In this study we considered how a common source of host heterogeneity in many species, the differences between the sexes in their capacity limit pathogen performance (see Table 1, Cousineau and Alizon 2014), can modify the expression of virulence in co-infections and the consequences this may have for the maintenance of genetic diversity in pathogen populations.

Our results indicate that the ability of co-infections to modify the relationship between pathogen growth (i.e. spore production) and pathogen induced reduction in host lifespan (i.e. virulence) will depend on the sex of the host. While infection in females is marked by a negative relationship between spore production and virulence, similar virulence patterns in male hosts correspond with more limited variation in spore production (Fig. 1). This was also observed in Thompson et al. (2017) where the scale of the trade-off between virulence and spore production varied due to host sex, driven largely by a reduction in overall variation in pathogen fitness across co-infection treatments in males. Despite this lower variation in spore production within male hosts, we found that the relative relationships between pathogen virulence and spore production were qualitatively similar between the sexes. Regardless of sex, simultaneous co-infections often exhibited relationships between pathogen virulence and spore production equal to that of the most virulent pathogen in isolation (Fig. 2, 3). Likewise, the relationship between virulence and spore production in sequential co-infections depended on pathogen genotype and order of establishment, but was unaffected by sex. These patterns culminate with females representing an environment within which pathogens can attain the highest levels of reproduction; yet coinfections within females also represent a substantial decrease in pathogen spore production as compared to the males. 
332 Despite commonalities between the sexes in the overall patterns of transmission and virulence,

333 the relative fitness between co-infecting pathogen genotypes varied strongly between males and

334 females. We found that females exhibited significant differences in spore production between

335 competing genotypes in all but one co-infection treatment, with the more virulent pathogen (C19)

336 producing up to 5.3 million spores more than its competitor (Simultaneous C19, C24; Fig. 4). In

337 contrast, when pathogens competed within males, each genotype produced an equal number of

338 transmission spores in simultaneous exposures and sequential exposures when the more virulent

339 pathogen established first (Fig. 4). Explaining these results may be that the more limited

exploitative environment of male Daphnia (Thompson et al. 2017; Gipson and Hall 2018) prohibits pathogens from exhibiting variation in exploitation strategies. Males then may be a reservoir for pathogen genetic diversity, resulting in equal fitness between competing genotypes and even

favouring genotypes which are frequently outcompeted within female hosts.

Our results also indicate that the arrival sequence of co-infecting pathogens will lead to different competitive outcomes depending on the host sex encountered. Previously established pathogens may inhibit later arriving competitors by blocking pathogen establishment, exhausting resources, or by inducing host immune responses (de Roode et al. 2005a; Lohr et al. 2010; Hoverman et al. 2013). In these situations, early establishing pathogens may exhibit considerable levels of reproduction even in competition with more virulent genotypes. Indeed, Ben-Ami et al. (2008) found that less virulent genotypes can exhibit substantial levels of reproduction when establishing first even though they are outcompeted when establishing after more virulent genotypes. Here, females exhibited this general pattern with the more virulent C19 genotype outcompeted by C24 and producing a similar number of spores as C1 when establishing second (Fig. 4). Conversely, the constraints imposed by male hosts appear to keep pathogen C19 from ever outcompeting other genotypes when they establish first. Consequentially, male hosts may thus maintain pathogen genetic variation that would otherwise be eroded by infection in females where the more virulent

Taken together, our results suggest that the evolutionary outcome of pathogen virulence in coinfection contexts will depend on how often pathogens encounter male and female hosts. In coinfections, more virulent pathogens often transmit more than their less virulent competitors, potentially favouring the evolution of higher virulence (de Roode et al. 2005b; Bell et al. 2006; Råberg et al. 2006; Ben-Ami et al. 2008; Balmer et al. 2009; Ben-Ami and Routtu 2013). Here, we find that co-infections in female hosts favour the transmission of the more virulent pathogen, 
potentially selecting for higher levels of virulence. In contrast, co-infection in males is characterized by either equal fitness of competing pathogens or higher fitness of less virulent pathogen genotypes. Co-infection in males may then either slow the evolution of virulence or completely reverse selection on virulence depending on the exposure context. Yet, the evolutionary outcome of virulence will depend not only on the within-host outcomes of infection, but also in how disease is transmitted between hosts (van Baalen and Sabelis 1995; Frank 1996; Mideo et al. 2008; Choisy and de Roode 2010; Alizon et al. 2013). Indeed, sex ratio can vary within a variety of species (Clutton-Brock and Iason 1986; Duneau and Ebert 2012 and Table 2 therein), including Daphnia (Galimov et al. 2011), which may influence the frequency of pathogen transmission between each sex and thus the overall patterns of selection on virulence. A higher proportion of the more exploitable sex, for example, may favour the transmission of more virulent pathogens, whereas population shifts to the less exploitable sex may restrain or reverse this pattern.

The ubiquity of pathogens in natural populations suggests that individuals are likely to encounter and become infected by multiple pathogen genotypes. We show that host sex may further impact on the relative fitness among pathogen genotypes due to within-host competition, finding that differences in the scale of the trade-off between pathogen virulence and transmission between the sexes affect the relative fitness among competing pathogens. This work reaffirms the role that host heterogeneity can play in affecting the outcome of within-host pathogen competition and its potential to influence the evolution of virulence (de Roode et al. 2004; Hodgson et al. 2004; Råberg et al. 2006; Ben-Ami et al. 2008; Ben-Ami and Routtu 2013; Izhar et al. 2015). Ultimately, how virulence should evolve within co-infection contexts will depend on frequency of encountering each sex as well as variation in the exploitative potential of male and female hosts. Collectively, our results suggest that the often observed relationship between virulence and pathogen competitive ability may not apply equally to each sex, providing a mechanism for the maintenance of pathogen genetic variation in sexually dimorphic host populations.

\section{Acknowledgements}

We would like to thank Isobel Booksmythe, Toby Hector, Louise Nørgaard, and Kyle Kelly for helpful conversations, laboratory assistance, and advice on statistical analysis. 
394 Table 1: Description of the four infection types used in this study. Single infections consisted of one genotype and co-infections consisted of 395 genotypes C19 and C24 or C19 and C1.

\begin{tabular}{|c|c|c|c|c|c|}
\hline \multirow{2}{*}{ Infection type } & \multirow{2}{*}{ Description } & \multicolumn{2}{|c|}{ Dose at 5 days old } & \multicolumn{2}{|c|}{ Dose at 12 days old } \\
\hline & & Geno. A & Geno. B & Geno. A & Geno. B \\
\hline Simultaneous single infection & $\begin{array}{l}\text { Individual was exposed once to a single } \\
\text { pathogen genotype }\end{array}$ & $\begin{array}{l}40,000 \\
\text { spores }\end{array}$ & - & - & - \\
\hline Simultaneous co-infection & $\begin{array}{l}\text { Individual was exposed once to two pathogen } \\
\text { genotypes }\end{array}$ & $\begin{array}{l}20,000 \\
\text { spores }\end{array}$ & $\begin{array}{l}20,000 \\
\text { spores }\end{array}$ & - & - \\
\hline Sequential single infection & $\begin{array}{l}\text { Individual was exposed to a pathogen at day } 5 \\
\text { and then to the same pathogen at day } 12 .\end{array}$ & $\begin{array}{l}20,000 \\
\text { spores }\end{array}$ & - & $\begin{array}{l}20,000 \\
\text { spores }\end{array}$ & - \\
\hline Sequential co-infection & $\begin{array}{l}\text { Individual was exposed to one pathogen at day } \\
5 \text { and then to a different pathogen at day } 12 \text {. }\end{array}$ & $\begin{array}{l}20,000 \\
\text { spores }\end{array}$ & - & - & $\begin{array}{l}20,000 \\
\text { spores }\end{array}$ \\
\hline
\end{tabular}


397 Table 2: Summary of univariate and multivariate analyses of variance describing the effects of host 398 sex, pathogen co-infection treatment, and their interaction on pathogen production of

399 transmission spores, pathogen induced reduction in host lifespan and their multivariate

400 interaction. Asterisks denote significant effects $(\alpha=0.05)$.

\begin{tabular}{|c|c|c|c|c|}
\hline Multivariate ANOVA & Pillai's trace & Approx. $F$ & d.f. & $p$-val \\
\hline \multicolumn{5}{|c|}{ Production of transmission spores, reduction in host lifespan (days) } \\
\hline Sex & 0.907 & 2939.443 & 2,604 & $<0.001 *$ \\
\hline Pathogen co-infection treatment & 0.167 & 5.027 & 22,1210 & $<0.001 *$ \\
\hline Sex : Pathogen & 0.108 & 3.133 & 22,1210 & $<0.001 *$ \\
\hline Univariate ANOVA & & $\boldsymbol{F}$ & d.f. & $p$-val \\
\hline \multicolumn{5}{|l|}{ Production of transmission spores } \\
\hline Sex & & 2048.260 & 1,605 & $<0.001 *$ \\
\hline Pathogen co-infection treatment & & 15.535 & 11,605 & $<0.001 *$ \\
\hline Sex : Pathogen & & 7.146 & 11,605 & $<0.001 *$ \\
\hline \multicolumn{5}{|l|}{ Reduction in host lifespan (days) } \\
\hline Sex & & 655.365 & 1,605 & $<0.001 *$ \\
\hline Pathogen co-infection treatment & & 6.110 & 11,605 & $<0.001 *$ \\
\hline Sex : Pathogen & & 0.526 & 11,605 & 0.886 \\
\hline
\end{tabular}


402 Table 3: Summary of an analyses of variance describing the effect of host sex, pathogen genotype, 403 and their interaction on relative pathogen fitness within co-infection treatments. Asterisks denote 404 significant effects $(\alpha=0.05)$.

\begin{tabular}{|c|c|c|c|}
\hline $\begin{array}{l}\text { Relative pathogen fitness } \\
\text { (relative spore production) }\end{array}$ & $\chi^{2}$ & d.f. & $p$-val \\
\hline \multicolumn{4}{|c|}{ Full model including all co-infection treatments } \\
\hline Sex & 170.335 & 1,574 & $<0.001 *$ \\
\hline Pathogen genotype & 49.093 & 1,574 & $<0.001 *$ \\
\hline Sex : Pathogen genotype & 69.034 & 1,574 & $<0.001 *$ \\
\hline \multicolumn{4}{|l|}{ Simultaneous C19, C24 } \\
\hline Sex & 40.511 & 1,72 & $<0.001 *$ \\
\hline Pathogen genotype & 57.442 & 1,72 & $<0.001 *$ \\
\hline Sex : Pathogen genotype & 43.890 & 1,72 & $<0.001 *$ \\
\hline \multicolumn{4}{|l|}{ Sequential C19, C24 } \\
\hline Sex & 47.780 & 1,98 & $<0.001 *$ \\
\hline Pathogen genotype & 48.988 & 1,98 & $<0.001 *$ \\
\hline Sex : Pathogen genotype & 49.133 & 1,98 & $<0.001 *$ \\
\hline \multicolumn{4}{|l|}{ Sequential C24, C19 } \\
\hline Sex & 26.541 & 1,88 & $<0.001 *$ \\
\hline Pathogen genotype & 6.991 & 1,88 & $0.008 *$ \\
\hline Sex: Pathogen genotype & 1.776 & 1,88 & 0.183 \\
\hline \multicolumn{4}{|l|}{ Simultaneous C19, C1 } \\
\hline Sex & 29.257 & 1,84 & $<0.001 *$ \\
\hline Pathogen genotype & 6.960 & 1,84 & $0.008 *$ \\
\hline Sex : Pathogen genotype & 5.386 & 1,84 & $0.020 *$ \\
\hline \multicolumn{4}{|l|}{ Sequential C19, C1 } \\
\hline Sex & 49.962 & 1,96 & $<0.001 *$ \\
\hline Pathogen genotype & 73.905 & 1,96 & $<0.001 *$ \\
\hline Sex : Pathogen genotype & 58.960 & 1,96 & $<0.001 *$ \\
\hline \multicolumn{4}{|l|}{ Sequential C1, C19 } \\
\hline Sex & 42.688 & 1,106 & $<0.001 *$ \\
\hline Pathogen genotype & 0.538 & 1,106 & 0.463 \\
\hline Sex : Pathogen genotype & 8.934 & 1,106 & $0.003 *$ \\
\hline
\end{tabular}


a.

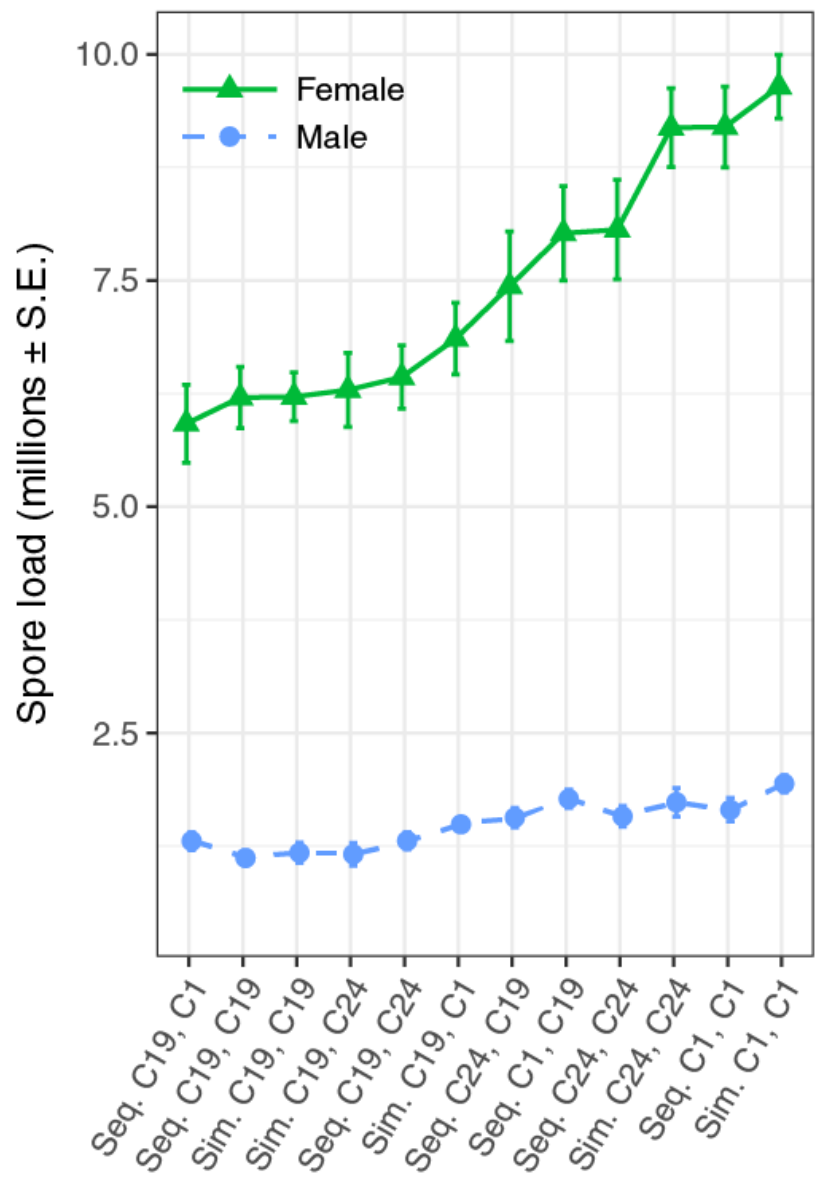

b.

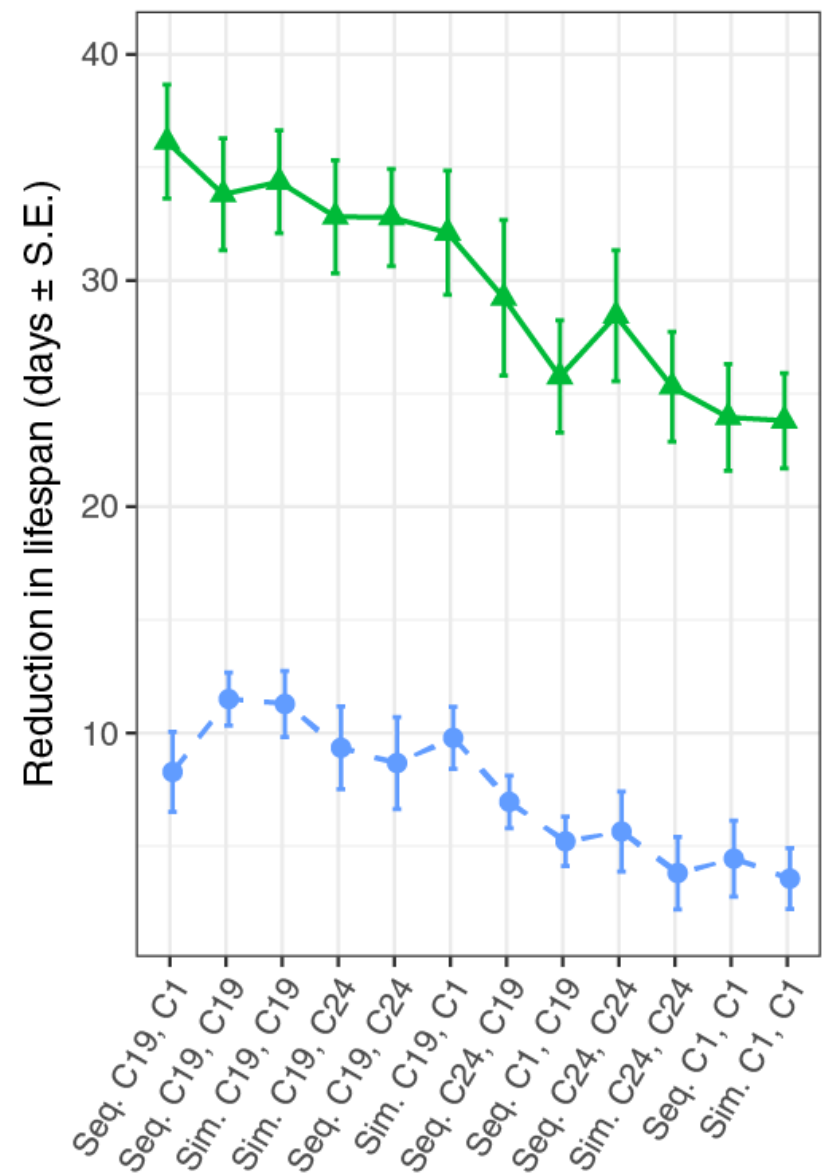

407 Figure 1: The influence of host sex and co-infection treatment on overall pathogen spore 408 production (a) and reduction in host lifespan (b). Shown are treatment means and standard errors. 409 Female results are represented by a solid green line with triangles and male results are 410 represented by a dashed blue line with circles. Pathogen co-infection treatments are ordered by 411 ascending average spore production in females. Sequential (Seq.) infection labels refer to the 412 order in which the two pathogen genotypes established in their hosts whereas simultaneous (Sim.) 413 infection labels refer to the two pathogen genotypes which established in their host at the same 414 time. 


\section{a. Females $-\mathrm{C} 19$ vs $\mathrm{C} 24$}

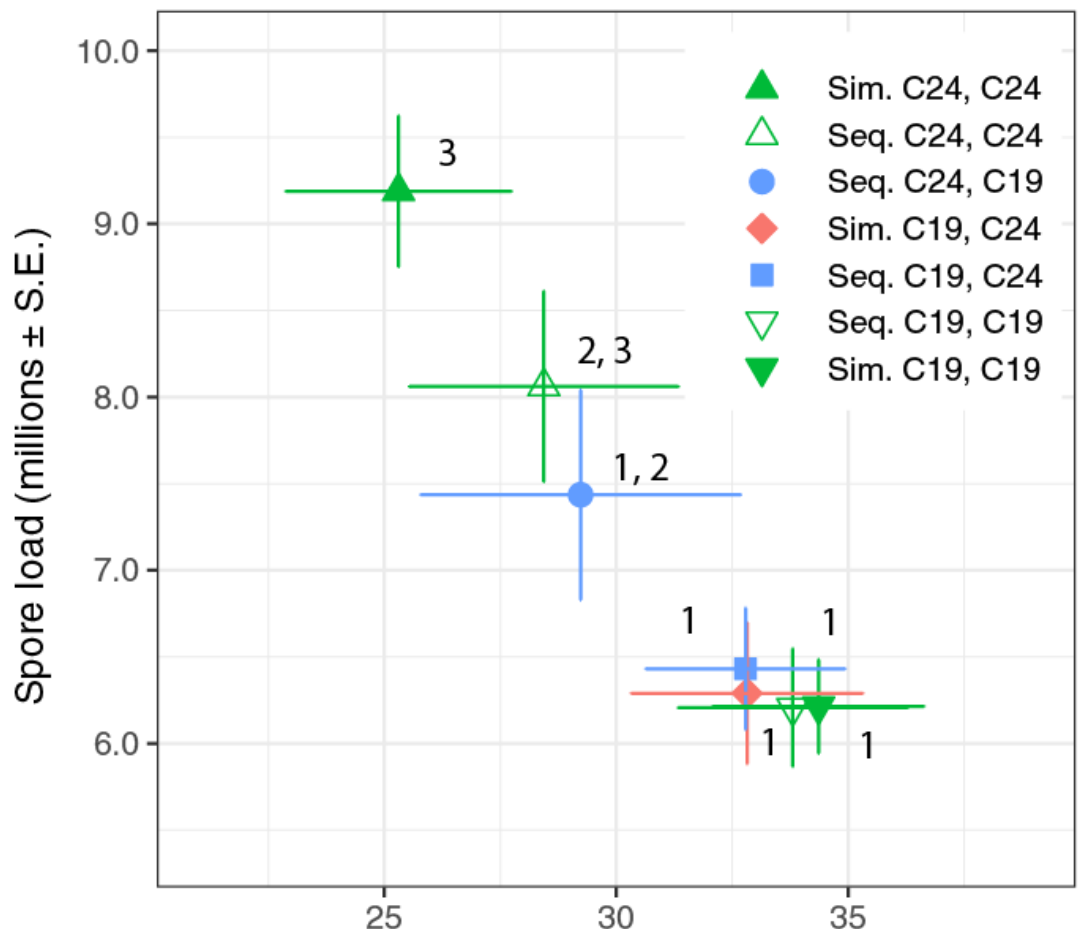

\section{b. Females $-\mathrm{C} 19$ vs $\mathrm{C} 1$}

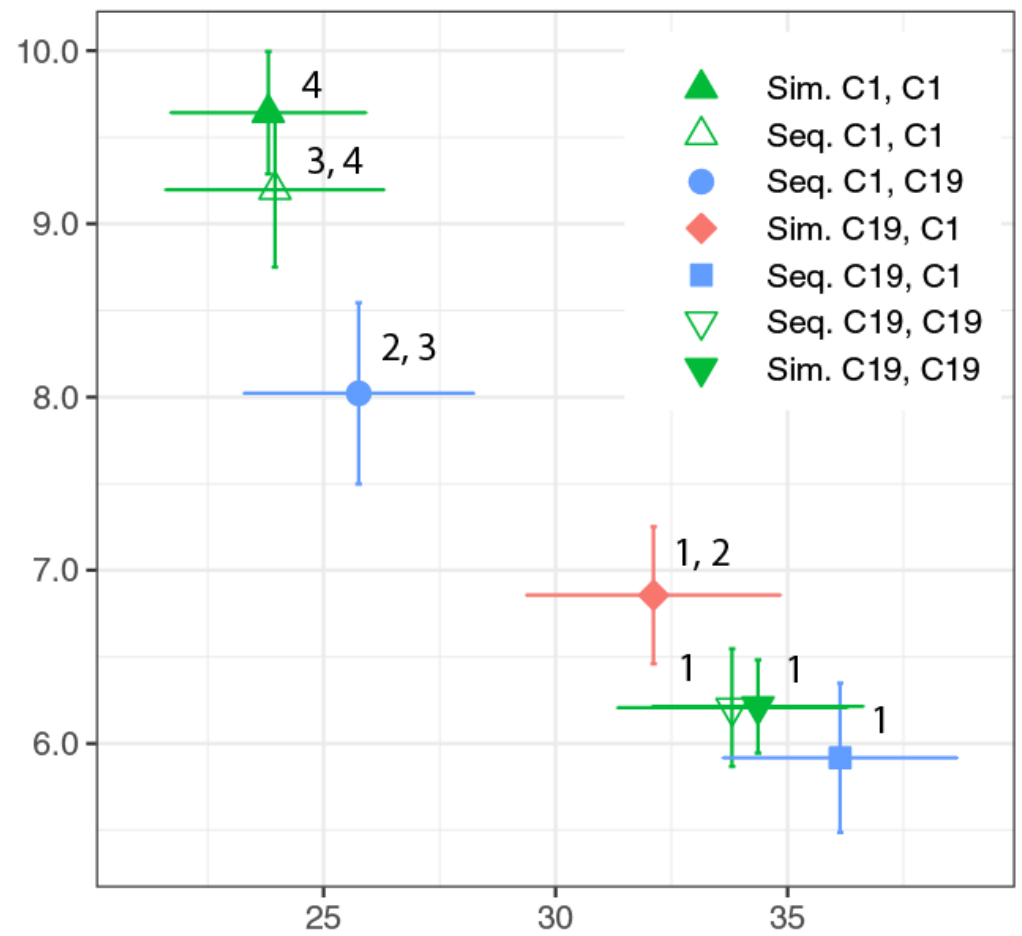

Virulence (days \pm S.E.)

416 Figure 2: The influence of co-infection treatment on the relationship between overall pathogen spore production and reduction in host lifespan

417 (virulence) for females exposed to pathogen genotype C19 and/or C24 (a) and females exposed to pathogen genotype C19 and/or C1 (b). Solid green

418 triangles indicate single simultaneous infections, open green triangles indicate single sequential infections, blue squares or circles refer to sequential

419 co-infections where the host was exposed to pathogen genotype C19 first or second respectively, and red diamonds refer to simultaneous co-

420 infections. Shown are multivariate treatment means and standard errors with different associated numbers signifying a significant difference in

421 multivariate mean between treatments. Sequential (Seq.) infection labels refer to the order in which the two pathogen genotypes established in

422 their hosts whereas simultaneous (Sim.) infection labels refer to the two pathogen genotypes which established in their host at the same time. 
a. Males - C19 vs C24

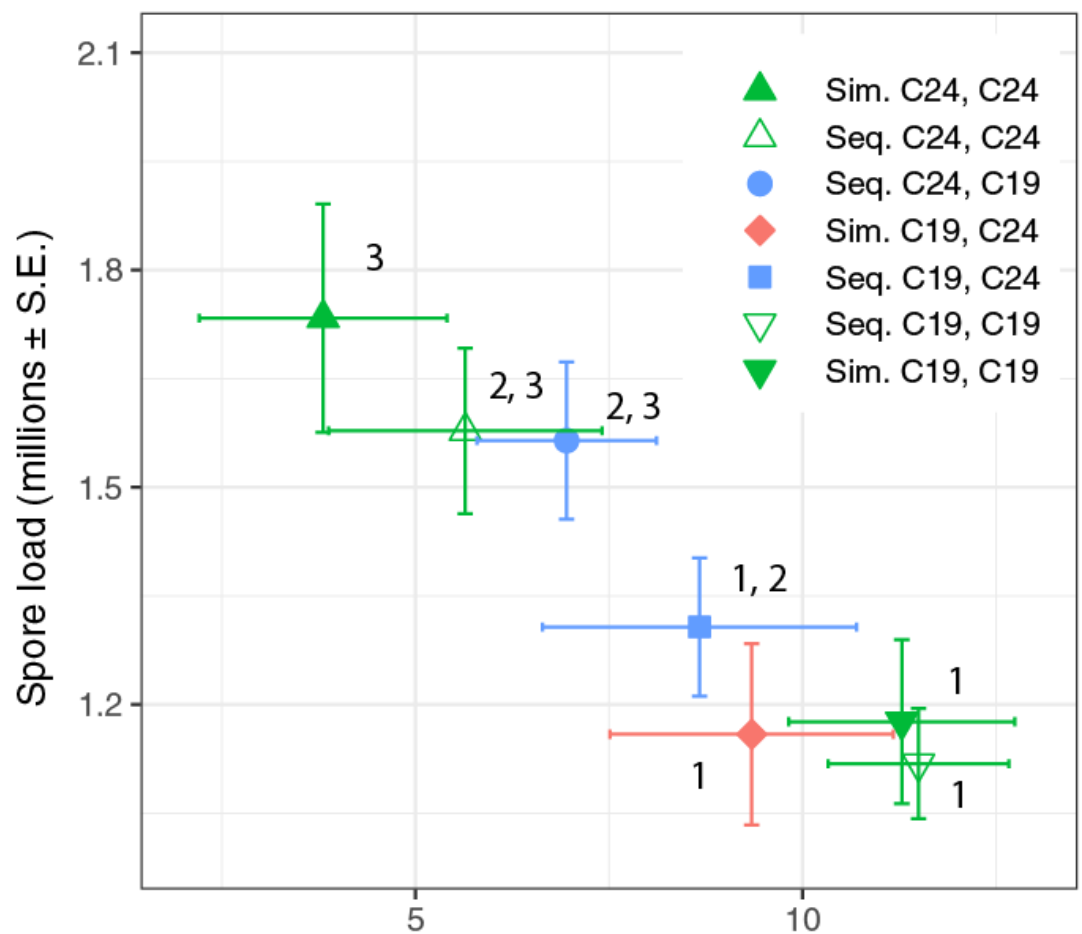

b. Males - C19 vs C1

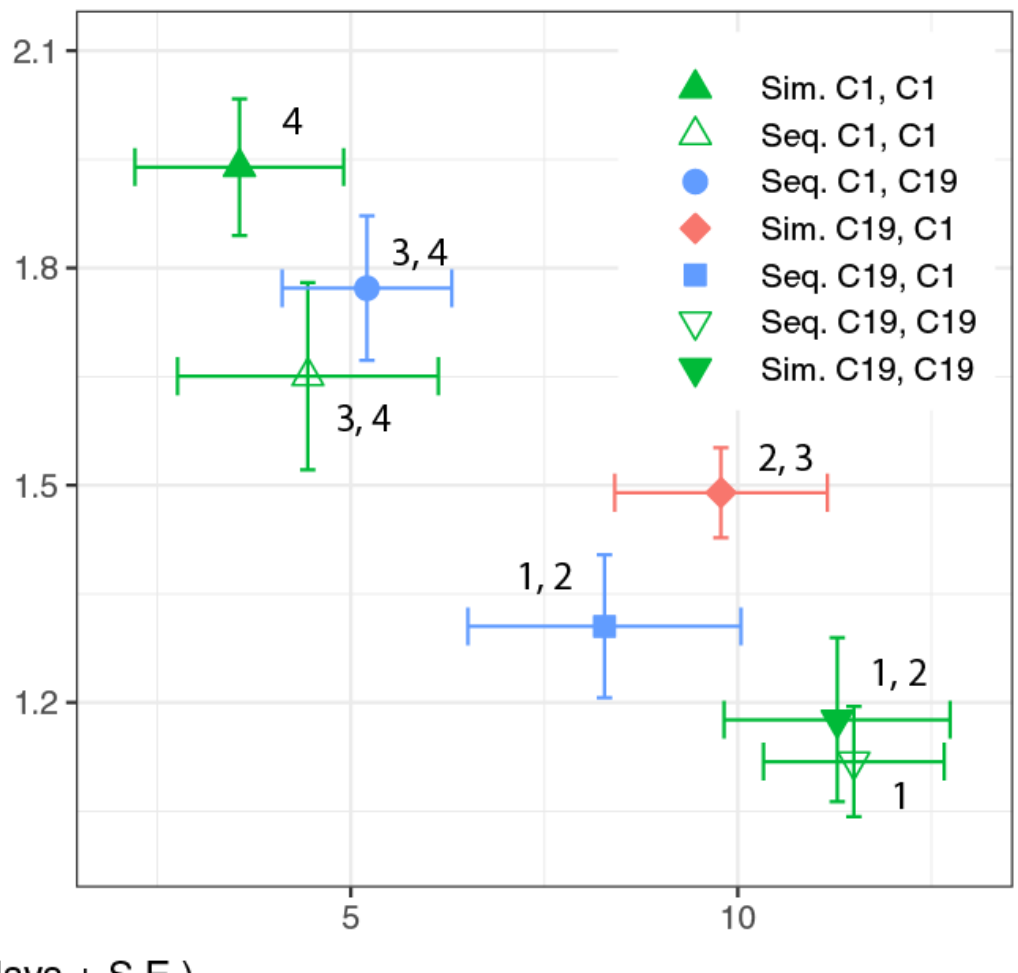

Virulence (days \pm S.E.)

424 Figure 3: The influence of co-infection treatment on the relationship between overall pathogen spore production and reduction in host lifespan 425 (virulence) for males exposed to pathogen genotype C19 and/or C24 (a) and males exposed to pathogen genotype C19 and/or C1 (b). Solid green 426 triangles indicate single simultaneous infections, open green triangles indicate single sequential infections, blue squares or circles refer to sequential co-infections where the host was exposed to pathogen genotype C19 first or second respectively, and red diamonds refer to simultaneous coinfections. Shown are multivariate treatment means and standard errors with different associated numbers signifying a significant difference in multivariate mean between treatments. Sequential (Seq.) infection labels refer to the order in which the two pathogen genotypes established in their hosts whereas simultaneous (Sim.) infection labels refer to the two pathogen genotypes which established in their host at the same time. 


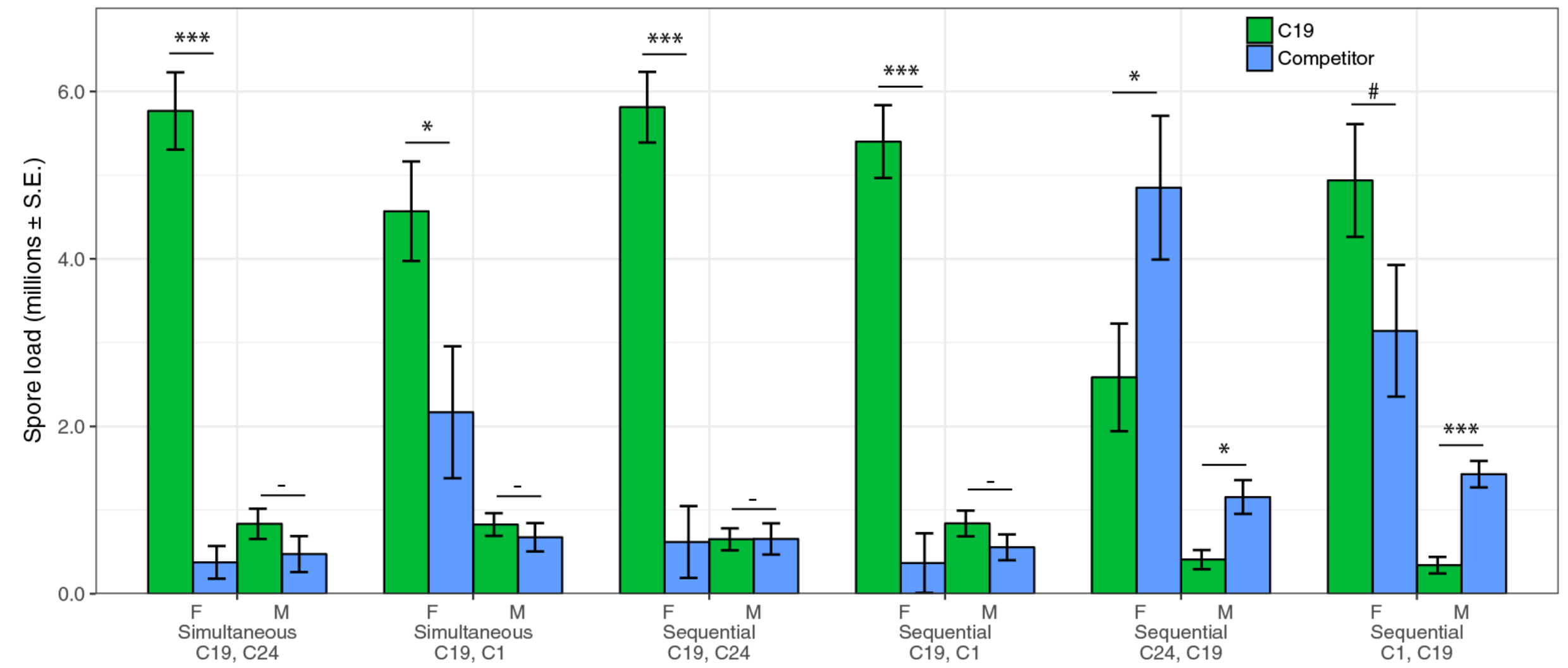

432 Figure 4: The influence of host sex and co-infection treatment on within-host pathogen competition as measured by relative spore production of 433 individual genotypes. Green bars represent spore production by pathogen genotype C19 and blue bars represent spore production by the competing 434 pathogen genotype (either C24 or C1). Shown are individual means for each genotype and standard errors. Asterisks indicate significant difference in mean spore production between competing genotypes within a single sex (two sample t-tests: *** $p<0.001, * * p<0.01, * p<0.05, \# p<0.10,->$ 


\section{References}

Alizon, S., J. C. de Roode, and Y. Michalakis. 2013. Multiple infections and the evolution of virulence. Ecol. Lett. 16:556-567.

Alizon, S., A. Hurford, N. Mideo, and M. Van Baalen. 2009. Virulence evolution and the trade-off hypothesis: History, current state of affairs and the future. J. Evol. Biol. 22:245-259.

Andras, J. P., and D. Ebert. 2013. A novel approach to parasite population genetics: Experimental infection reveals geographic differentiation, recombination and host-mediated population structure in Pasteuria ramosa, a bacterial parasite of Daphnia. Mol. Ecol. 22:972-86.

Balmer, O., S. C. Stearns, A. Schötzau, and R. Brun. 2009. Intraspecific competition between coinfecting parasite strains enhances host survival in African trypanosomes. Ecology 90:3367-3378.

Balmer, O., and M. Tanner. 2011. Prevalence and implications of multiple-strain infections. Lancet Infect. Dis. 11:868-878. Elsevier Ltd.

Bashey, F. 2015. Within-host competitive interactions as a mechanism for the maintenance of parasite diversity. Philos. Trans. R. Soc. B 370:20140301.

Bates, D., M. Maechler, B. Bolker, and S. Walker. 2015. Fitting linear mixed-effects models using Ime4. J. Stat. Softw. 67:1-48.

Bell, A. S., J. C. de Roode, D. Sim, and A. F. Read. 2006. Within-host competition in genetically diverse malaria infections: parasite virulence and competitive success. Evolution 60:1358-1371.

Ben-Ami, F., L. Mouton, and D. Ebert. 2008. The effects of multiple infections on the expression and evolution of virulence in a Daphnia-endoparasite system. Evolution 62:1700-1711.

Ben-Ami, F., and J. Routtu. 2013. The expression and evolution of virulence in multiple infections: the role of specificity, relative virulence and relative dose. BMC Evol. Biol. 13:1. BMC Evolutionary Biology.

Bull, J. J. 1994. Virulence. Evolution 48:1423-1437.

Bussière, L. F., M. Demont, A. J. Pemberton, M. D. Hall, and P. I. Ward. 2010. The assessment of insemination success in yellow dung flies using competitive PCR. Mol. Ecol. Resour. 10:292-303. 
Chao, L., K. A. Hanley, C. L. Burch, C. Dahlberg, and P. E. Turner. 2000. Kin selection and parasite evolution: higher and lower virulence with hard and soft selection. Q. Rev. Biol. 75:261-275.

Choisy, M., and J. C. de Roode. 2010. Mixed infections and the evolution of virulence: effects of resource competition, parasite plasticity, and impaired host immunity. Am. Nat. 175:E105-E118.

Christe, P., O. Glaizot, G. Evanno, N. Bruyndonckx, G. Devevey, G. Yannic, P. Patthey, A. Maeder, P. Vogel, and R. Arlettaz. 2007. Host sex and ectoparasites choice: Preference for, and higher survival on female hosts. J. Anim. Ecol. 76:703-710.

Clerc, M., D. Ebert, and M. D. Hall. 2015. Expression of parasite genetic variation changes over the course of infection: implications of within-host dynamics for the evolution of virulence. Proc. R. Soc. Lond. B. 282.

Clutton-Brock, T. H., and G. R. Iason. 1986. Sex ratio variation in mammals. Q. Rev. Biol. 61:339374.

Cousineau, S. V., and S. Alizon. 2014. Parasite evolution in response to sex-based host heterogeneity in resistance and tolerance. J. Evol. Biol. 27:2753-2766.

Cressler, C. E., D. V. McLeod, C. Rozins, J. Van Den Hoogen, and T. Day. 2016. The adaptive evolution of virulence: a review of theoretical predictions and empirical tests. Parasitology 143:915-930.

de Roode, J. C., R. Culleton, S. J. Cheesman, R. Carter, and A. F. Read. 2004. Host heterogeneity is a determinant of competitive exclusion or coexistence in genetically diverse malaria infections. Proc. R. Soc. B Biol. Sci. 271:1073-1080.

de Roode, J. C., M. E. H. Helinski, M. A. Anwar, and A. F. Read. 2005a. Dynamics of multiple infection and within-host competition in genetically diverse malaria infections. Am. Nat. 166:531542.

de Roode, J. C., R. Pansini, S. J. Cheesman, M. E. H. Helinski, S. Huijben, A. R. Wargo, A. S. Bell, B. H. K. Chan, D. Walliker, and A. F. Read. 2005b. Virulence and competitive ability in genetically diverse malaria infections. Proc. Natl. Acad. Sci. U. S. A. 102:7624-8.

Duneau, D., and D. Ebert. 2012. Host sexual dimorphism and parasite adaptation. PLoS Biol. 
10:e1001271.

Duneau, D., P. Luijckx, L. F. Ruder, and D. Ebert. 2012. Sex-specific effects of a parasite evolving in a female-biased host population. BMC Biol. 10:104.

Ebert, D. 2005. Ecology, epidemiology, and evolution of parasitism in Daphnia. 1st ed. National Library of Medicine (US), National Center for Biotechnology Information.

http://www.ncbi.nlm.nih.gov/entrez/query.fcgi?db=Books, Bethesda, MD.

Ebert, D., D. Duneau, M. D. Hall, P. Luijckx, J. P. Andras, L. Du Pasquier, and F. Ben-Ami. 2016. A population biology perspective on the stepwise infection process of the bacterial pathogen Pasteuria ramosa in Daphnia. Adv. Parasitol. 91:265-310.

Ebert, D., C. D. Zschokke-Rohringer, and H. J. Carius. 1998. Within-and between-population variation for resistance of Daphnia magna to the bacterial endoparasite Pasteuria ramosa. Proc. R. Soc. London. Ser. B Biol. Sci. 265:2127-2134.

Eswarappa, S. M., S. Estrela, and S. P. Brown. 2012. Within-host dynamics of multi-species infections: Facilitation, competition and virulence. PLoS One 7.

Fox, J., and S. Weisberg. 2011. An $\{$ R $\}$ Companion to Applied Regression. Second. Sage, Thousand Oaks, CA.

Frank, S. A. 1996. Models of parasite virulence. Q. Rev. Biol. 71:37-78.

Galimov, Y., B. Walser, and C. R. Haag. 2011. Frequency and inheritance of non-male producing clones in Daphnia magna: evolution towards sex specialization in a cyclical parthenogen? J. Evol. Biol. 24:1572-1583.

Giefing-Kröll, C., P. Berger, G. Lepperdinger, and B. Grubeck-Loebenstein. 2015. How sex and age affect immune responses, susceptibility to infections, and response to vaccination. Aging Cell 309321.

Gipson, S. A. Y., and M. D. Hall. 2018. Interactions between host sex and age of exposure modify the virulence-transmission trade-off. J. Evol. Biol., doi: 10.1111/jeb.13237.

Gipson, S. A. Y., and M. D. Hall. 2016. The evolution of sexual dimorphism and its potential impact on host-pathogen coevolution. Evolution 70:959-968. 
Hodgson, D. J., R. B. Hitchman, A. J. Vanbergen, R. S. Hails, R. D. Possee, and J. S. Cory. 2004. Host ecology determines the relative fitness of virus genotypes in mixed-genotype nucleopolyhedrovirus infections. J. Evol. Biol. 17:1018-1025.

Hoverman, J. T., B. J. Hoye, and P. T. J. Johnson. 2013. Does timing matter? How priority effects influence the outcome of parasite interactions within hosts. Oecologia 173:1471-1480.

Izhar, R., J. Routtu, and F. Ben-ami. 2015. Host age modulates within-host parasite competition. Biol. Lett. 11.

Klein, S. L., and K. L. Flanagan. 2016. Sex differences in immune responses. Nat. Rev. Immunol., doi: $10.1038 /$ nri.2016.90.

Klüttgen, B., U. Dülmer, M. Engels, and H. T. Ratte. 1994. ADaM, an artificial freshwater for the culture of zooplankton. Water Res. 28:743-746.

Lohr, J. N., M. Yin, and J. Wolinska. 2010. Prior residency does not always pay off - co-infections in Daphnia. Parasitology 137:1493-1500.

Louhi, K.-R., L.-R. Sundberg, J. Jokela, and A. Karvonen. 2015. Interactions among bacterial strains and fluke genotypes shape virulence of co-infection. Proc. R. Soc. B 282:20152097.

Luijckx, P. 2012. Host specificity and genetics of host resistance in the Daphnia-Pasteuria hostparasite system.

Massey, R. C., A. Buckling, and R. ffrench-Constant. 2004. Interference competition and parasite virulence. Proc. R. Soc. B Biol. Sci. 271:785-788.

McCurdy, D. G., D. Shutler, A. Mullie, and M. R. Forbes. 1998. Sex-biased parasitism of avian hosts: relations to blood parasite taxon and mating system. Oikos 82:303-312.

Mideo, N. 2009. Parasite adaptations to within-host competition. Trends Parasitol. 25:261-268.

Mideo, N., S. Alizon, and T. Day. 2008. Linking within- and between-host dynamics in the evolutionary epidemiology of infectious diseases. Trends Ecol. Evol. 23:511-517.

Mouton, L., G. Nong, J. F. Preston, and D. Ebert. 2007. Variable-number tandem repeats as molecular markers for biotypes of Pasteuria ramosa in Daphnia spp. Appl. Environ. Microbiol. 
73:3715-3718.

Parker, G. A. 2006. Sexual conflict over mating and fertilization: an overview. Philos. Trans. R. Soc. Lond. B. Biol. Sci. 361:235-259.

Poulin, R. 1996. Sexual inequalities in helminth infections: a cost of being a male. Am. Nat. 147:287-295.

Råberg, L., J. C. de Roode, A. S. Bell, P. Stamou, D. Gray, and A. F. Read. 2006. The role of immunemediated apparent competition in genetically diverse malaria infections. Am. Nat. 168:41-53.

Read, A. F., and L. H. Taylor. 2001. The ecology of genetically diverse infections. Science 292:10991102.

Rigaud, T., M.-J. Perrot-Minnot, and M. J. F. Brown. 2010. Parasite and host assemblages: embracing the reality will improve our knowledge of parasite transmission and virulence. Proc. R. Soc. B Biol. Sci. 277:3693-3702.

Rolff, J. 2002. Bateman's principle and immunity. Proc. Biol. Sci. 269:867-72.

Schalk, G., and M. R. Forbes. 1997. Male biases in parasitism of mammals: effects of study type, host age, and parasite taxon. Oikos 78:67-74.

Schärer, L., L. Rowe, and G. Arnqvist. 2012. Anisogamy, chance and the evolution of sex roles. Trends Ecol. Evol. 27:260-264.

Sheridan, L. a D., R. Poulin, D. F. Ward, and M. Zuk. 2000. Sex differences in parasitic infections among arthropod hosts: is there a male bias? Oikos 88:327-334.

Thompson, O., S. A. Y. Gipson, and M. D. Hall. 2017. The impact of host sex on the outcome of coinfection. Sci. Rep. 7:1-7. Springer US.

van Baalen, M., and M. W. Sabelis. 1995. The Dynamics of Multiple Infection and the Evolution of Virulence. Am. Nat. 146:881-910.

Wolinska, J., and K. C. King. 2009. Environment can alter selection in host-parasite interactions. Trends Parasitol. 25:236-244.

Zuk, M. 2009. The sicker sex. PLoS Pathog. 5:e1000267. 
bioRxiv preprint doi: https://doi.org/10.1101/306985; this version posted April 25, 2018. The copyright holder for this preprint (which was not certified by peer review) is the author/funder, who has granted bioRxiv a license to display the preprint in perpetuity. It is made available under aCC-BY-NC-ND 4.0 International license. 